\title{
STUDY OF RELATIONSHIP BETWEEN THE PROTEIN OXIDATION MARKERS AND ADIPOKINES IN OBESE TYPE 2 DIABETIC PATIENTS
}

\author{
SUKHPAL SINGH ${ }^{1,2}$, AMITA MAHAJAN ${ }^{2,3}$, JASPREET KAUR $^{1 *}$ \\ ${ }^{1}$ Department of Biotechnology, University Institute of Engineering and Technology, Panjab University, Chandigarh, India. ${ }^{2}$ Department \\ of Medical Lab Technology, I. K. Gujral Punjab Technical University, Jalandhar, Punjab, India. ${ }^{3}$ University School of Medical and Allied \\ Sciences, Rayat Bahra University, Mohali, Punjab, India. Email:jaspreet_uiet@pu.ac.in
}

Received: 29 March 2019, Revised and Accepted: 29 April 2019

ABSTRACT

Objective: This study was done to explore the correlation if any, between obesity markers adiponectin, leptin, and protein oxidative stress (OS) status in obese with and without type 2 diabetic mellitus (T2DM) patients.

Methods: In the present study, 30 healthy subjects, 30 obese non-diabetics, and 30 obese T2DM patients were enrolled. Protein OS parameters such as advanced oxidation of protein products (AOPPs) and protein carbonyl (PC) were estimated. Serum leptin, adiponectin, and insulin levels were measured by ELISA.

Results: The AOPP, PC, leptin, leptin adiponectin ratio (LAR), insulin, and homeostasis model assessment of insulin resistance (HOMA-IR) levels were significantly higher in obese non-diabetic and obese T2DM as compared to healthy control ( $<<0.001)$. However, serum adiponectin levels were significantly lower in obese non-diabetic and obese T2DM as compared to control ( $\mathrm{p}<0.001)$. HOMA-IR and LAR both the index of IR were increased in obese non-diabetic and obese T2DM. Positive correlations were observed for AOPP with body mass index, PC in obese non-diabetic and with fasting blood glucose, postprandial blood glucose, HOMA-IR, and PC in obese T2DM. A negative correlation was found between PC and adiponectin in obese non-diabetic and obese T2DM. A significant inverse correlation was obtained between leptin and adiponectin in obese T2DM only.

Conclusion: The present study suggested that increased protein OS, hyperinsulinemia, and hyperleptinemia may lead to hypoadiponectinemia in obese with and without T2DM. Moreover, determination of protein oxidation markers can be useful for monitoring the dysregulation of adipokines and glucose metabolism in obesity and T2DM.

Keywords: Obesity, Type 2 diabetes, Adiponectin, Leptin, Protein carbonyl.

(c) 2019 The Authors. Published by Innovare Academic Sciences Pvt Ltd. This is an open access article under the CC BY license (http://creativecommons. org/licenses/by/4. 0/) DOI: http://dx.doi.org/10.22159/ajpcr.2019.v12i6.33280

\section{INTRODUCTION}

Obesity is an epidemic and worldwide complication that represents a major health issue of the $21^{\text {st }}$ century. Many studies have shown that obesity, especially visceral obesity is linked to chronic low-grade inflammation in hypertrophied adipose tissue that leads to initiation and progression of metabolic disorders such as dyslipidemia, hypertension, atherosclerosis, insulin resistance (IR), and type 2 diabetic mellitus (T2DM) [1]. Adipose tissue is not only a major source of energy for the human body but also inert storage reservoir for lipids [2]. The term adipokines can be defined as a biologically active substance released by adipose tissue [3]. It is now considered as a largest endocrine organ of the body that helps in the regulation of glucose and lipid metabolism, insulin sensitivity and secretion, inflammatory process, appetite, satiety, energy expenditure, endothelial function, blood pressure, and neuroendocrine regulation [4]. Most of the adipokines are proinflammatory and less the number of anti-inflammatory adipokines $[5,6]$.

Adiponectin is an anti-inflammatory $30 \mathrm{kDa}$ plasma protein and abundantly expressed in white adipose tissue [7]. A large number of experimental studies on rodent and cell culture model have demonstrated that adiponectin has a protective role in cardiovascular diseases [8]. In human studies decreased circulating levels of adiponectin in patients with cardiovascular disease give support to these findings [9]. A decrease in the level of adiponectin has been associated with IR, dyslipidemia, and atherosclerosis in human and rodents $[6,9]$. It has a protective role against metabolic diseases and promotes insulin sensitivity [10]. It also maintains and widespread healthy adipose tissue by preventing ectopic lipid accumulation in an animal model and enhances insulin sensitivity $[9,11]$.
In contrast, leptin is proinflammatory, a product of obese $(o b)$ gene is a $16-\mathrm{kDa}$ circulating protein hormone produced by adipocytes. It is an adipokines and a key molecule that regulates food intake and body weight [12]. The level of plasma leptin is directly proportional to the mass of body fat [13]. It also plays an important role in the regulation of body metabolism, energy homeostasis, and neuroendocrine function [14]. It acts by binding to circulating receptors, triggers Janus kinase, and induces signal transducer and activator of transcription pathway [15]. Increased leptin directly modulates pancreatic beta cells functions and indirectly through central neural pathways leading to progression of IR and T2DM [4,16]. Dysregulation of adipokines occurs through the generation of reactive oxygen species (ROS) in adipose tissue induces systemic oxidative stress (OS) in obesity. Moreover, the following OS is, directly and indirectly, related to the pathogenesis of obesity-associated IR [17].

Advanced oxidation of protein products (AOPPs) are ROS generated chloraminated oxidants, mainly hypochlorous acid and chloramines, derived by myeloperoxidase in activated neutrophils [18]. They are defined as dityrosine-containing cross-linked protein products and are a reliable marker to measure the degree of protein oxidation $[19,20]$, whereas determination of plasma protein carbonyl (PC) is most stable and commonly used as an early marker of protein oxidation $[21,22]$. Increased level of carbonyl content has been found in several diseases such as rheumatoid arthritis, Alzheimer's disease, diabetic mellitus, and muscle damage due to strenuous exercise. With the binding of metal cations to protein and further attack of ROS, especially hydrogen peroxide and oxygen species modify amino acid lead to the formation 
of carbonyls [23]. Increased level of AOPP and PC has been found in obesity and diabetes $[20,23]$.

From extensive literature study, we found that there is a lack of data to clearly explain the relationship between circulating metabolic homeostasis adipokines and protein OS parameters in North India population specifically from Chandigarh region and its surroundings. This study evaluates the comparative and relative relationship between leptin, adiponectin, leptin adiponectin ratio (LAR), insulin, homeostasis model assessment of IR (HOMA-IR), and protein OS markers in obese subjects as well as in obese T2DM patients.

\section{MATERIALS AND METHODS}

This case-control study included 90 subjects, of which 30 healthy controls (body mass index [BMI] $\leq 25 \mathrm{~kg} / \mathrm{m}^{2}$ ), 30 obese non-diabetics $\left(\mathrm{BMI} \geq 30 \mathrm{~kg} / \mathrm{m}^{2}\right)$, and 30 obese T2DM patients (BMI $\geq 30 \mathrm{~kg} / \mathrm{m}^{2}$ ) were enrolled.

\section{Anthropometric parameters and subject selection}

The anthropometric parameters included weight and height measurements for determination of BMI. Each subject was provided a questionnaire for information about age, sex, height, weight, smoking status, and alcohol consumption. Care was taken to select healthy control subjects with BMI $\left(\leq 25 \mathrm{~kg} / \mathrm{m}^{2}\right)$ and had no family history of diabetes mellitus. The inclusion criteria for age in all groups were $30-50$ years. For diabetic patients duration of diabetes was at least 5 years or more, fasting glucose $\geq 126 \mathrm{mg} / \mathrm{dl}$ and level of glycated hemoglobin (HbA1C) $\geq 6.5 \%$. The criteria for selection of type 2 diabetic patients were in accordance with the World Health Organization, and all were under diabetic treatment with oral hypoglycemic drugs. This study excluded the patients taking antioxidants supplements, anti-inflammatory drugs, antiobesity therapies, inflammatory or malignant disease, history of hospitalization in preceding 6 months, pregnant and lactating women, Type I diabetic patients, and exogenous insulin. The study was approved by the Government Multispecialty hospital, Chandigarh
(MS-II-2015/4631), India. All the participants were informed about the objectives of the study and written informed consent was obtained from all subjects involved in this study.

\section{Sample collection}

Venous peripheral blood sample of each subject was collected after 10-12 h of overnight fasting. Samples were collected into plain sterile and ethylenediaminetetraacetic acid (EDTA) anticoagulant Vacutainers tubes. For estimation of $\mathrm{HbA1C}$ whole blood collected in EDTA was used. Plasma samples were used for the measurement of AOPP and PC. The fasting serum samples were used for the measurement of fasting blood glucose (FBG), lipid profile, insulin, leptin, and adiponectin. Serum and plasma were stored at $-80^{\circ} \mathrm{C}$ until further use.

\section{Biochemical measurements}

HbA1C was measured using commercially available kits (Erba Diagnostics Mannheim, Germany) by ion exchange resin method. Serum leptin and insulin level were measured quantitatively using solid phase enzyme-linked immunosorbent assay ELISA kit (DRG International, Inc., USA) and serum adiponectin was measured by human Acpro 30 ELISA kit (Ray Biotech Inc., USA). AOPP level was measured by the spectrophotometric method of Witko-Sarsat et al. 1998 [18]. PC level was measured by the method of Reznick and Packer 1994 [21]. Blood glucose, total cholesterol (TC), triglycerides (TG), and high-density lipoprotein (HDL) cholesterol were measured by Erba Transasia diagnostic kits. Low-density lipoprotein (LDL) cholesterol and very LDL (VLDL) cholesterol were calculated indirectly by Friedewald's formula [24]. IR was calculated by the HOMA-IR [25].

HOMA-IR = Fasting insulin $(\mu \mathrm{U} / \mathrm{ml}) \times$ Fasting glucose $(\mathrm{mmol} / \mathrm{L}) / 22.5$

\section{Statistical analysis}

IBM SPSS statistics 25.0.0 was used for data analysis. Data (quantitative) variable were presented as a mean \pm standard deviation. Parametric one-way ANOVA for normally distributed variables to compared between groups followed by post hoc test. Nonparametric Kruskal-

Table 1: Clinical and demographical details of the subjects in groups

\begin{tabular}{|c|c|c|c|}
\hline \multirow[t]{2}{*}{ Parameters } & Healthy control & Obese non-diabetic & Obese T2DM \\
\hline & $\mathrm{BMI} \leq 25$ & $\mathrm{BMI} \geq 30$ & $\mathrm{BMI} \geq 30$ \\
\hline $\mathrm{n}$ & 30 & 30 & 30 \\
\hline Age (years) & $40.23 \pm 6.89$ & $41.30 \pm 6.48$ & $47.40 \pm 3.50$ \\
\hline $\mathrm{p}$ & & $<0.504^{*}$ & $<0.001^{*},<0.001^{* *} 8$ \\
\hline Gender (male/female) & $18 / 12$ & $20 / 10$ & $15 / 15$ \\
\hline BMI $\left(\mathrm{kg} / \mathrm{m}^{2}\right)$ & $22.67 \pm 2.04$ & $32.63 \pm 2.73$ & $32.83 \pm 2.56$ \\
\hline $\mathrm{p}$ & & $<0.001^{*}$ & $<0.001^{*},<0.464^{* *}$ \\
\hline FBG $(\mathrm{mmol} / \mathrm{L})$ & $4.47 \pm 0.20$ & $4.64 \pm 0.35$ & $10.47 \pm 0.77$ \\
\hline $\mathrm{p}$ & & $<0.026^{*}$ & $<0.001^{*},<0.001^{* *}$ \\
\hline PPBG (mmol/L) & $6.37 \pm 0.71$ & $6.75 \pm 0.69$ & $14.91 \pm 2.54$ \\
\hline $\mathrm{p}$ & & $<0.617^{*}$ & $<0.001^{*},<0.001^{* *}$ \\
\hline HbA1c (\%) & $4.54 \pm 0.36$ & $4.60 \pm 0.38$ & $8.92 \pm 0.96$ \\
\hline $\mathrm{p}$ & & $<0.908^{*}$ & $<0.001^{*},<0.001^{* *}$ \\
\hline Cholesterol (mg/dl) & $156.47 \pm 22.55$ & $184.57 \pm 29.45$ & $195.17 \pm 32.17$ \\
\hline $\mathrm{p}$ & & $<0.001^{*}$ & $<0.001^{*},<0.204^{* *}$ \\
\hline Triglycerides (mg/dl) & $110.37 \pm 32.31$ & $167.01 \pm 45.00$ & $246.89 \pm 48.55$ \\
\hline $\mathrm{p}$ & & $<0.001^{*}$ & $<0.001^{*},<0.001^{* *}$ \\
\hline HDL-cholesterol (mg/dl) & $51.23 \pm 5.32$ & $48.34 \pm 5.16$ & $39.85 \pm 4.42$ \\
\hline $\mathrm{p}$ & & $<0.069$ & $<0.001^{*},<0.001^{* *}$ \\
\hline LDL-cholesterol (mg/dl) & $83.17 \pm 19.40$ & $102.83 \pm 31.63$ & $105.94 \pm 32.57$ \\
\hline $\mathrm{p}$ & & $<0.011^{*}$ & $<0.003^{*},<0.756^{* *}$ \\
\hline VLDL-cholesterol (mg/dl) & $22.07 \pm 6.46$ & $33.40 \pm 9.00$ & $49.38 \pm 9.71$ \\
\hline $\mathrm{p}$ & & $<0.001^{*}$ & $<0.001^{*},<0.001^{* *}$ \\
\hline Insulin $(\mu \mathrm{IU} / \mathrm{ml})$ & $6.78 \pm 2.01$ & $13.17 \pm 4.31$ & $13.58 \pm 3.85$ \\
\hline $\mathrm{p}$ & & $<0.001^{*}$ & $<0.001^{*},<0.728^{* *}$ \\
\hline HOMA-IR & $1.35 \pm 0.42$ & $2.74 \pm 0.99$ & $6.17 \pm 1.72$ \\
\hline $\mathrm{p}$ & & $<0.001^{*}$ & $<0.001^{*},<0.001^{* *}$ \\
\hline
\end{tabular}

Data are represented as mean \pm SD. p $<0.05$ statistically significant. ${ }^{*}$ p significance versus Healthy control, ${ }^{* *}$ p significance versus obese nondiabetic. BMI: Body mass index, FBG: Fasting blood glucose, PPBG: Post-prandial blood glucose, T2DM: Type 2 diabetes mellitus, HDL: High-density lipoprotein, LDL: Low-density lipoprotein, HOMA-IR: Homeostasis model assessment of insulin, HbA1c: Glycated hemoglobin, SD: Standard deviation 
Wallis followed by Mann-Whitney U-tests for not normally distributed variables. Correlation analysis between variables for all groups was analyzed by Spearman's correlation test. p<0.05 (two-tailed) was studied statistically significant.

\section{RESULTS}

The demographical and clinical parameters studied in all groups were classified by BMI

In Table 1, FBG, TC, TG, LDL-cholesterol, VLDL-cholesterol, insulin, and HOMA-IR were found to be with significantly higher in obese-non diabetic and obese T2DM groups as compared to healthy control $(\mathrm{p}<0.05$ or $\mathrm{p}<0.001)$. Age, postprandial blood glucose (PPBG), HDL-cholesterol, and HbA1c significantly higher in obese T2DM as compared to obese non-diabetic and healthy control $(\mathrm{p}<0.001)$. In Table 2 , leptin showed significantly increased whereas adiponectin significantly decreased in both obese non-diabetic and obese T2DM as compared to healthy control $(\mathrm{p}<0.001)$. LAR, AOPP, and PC significantly higher in obese non-diabetic and obese T2DM groups as compared to healthy control $(\mathrm{p}<0.001)$.

Correlation analysis studied among different parameters in all groups

In healthy control, AOPP showed positive correlation with $\mathrm{HbA1c}$ $(\mathrm{r}=0.411, \mathrm{p}<0.024)$, between insulin and HOMA-IR $(\mathrm{r}=0.977, \mathrm{p}<0.001)$. LAR showed positive correlation with leptin $(r=0.914, p<0.001)$ but negative correlation with adiponectin $(\mathrm{r}=-0.547, \mathrm{p}<0.002)$ and $\mathrm{HbA1c}$ $(\mathrm{r}=-0.383, \mathrm{p}<0.037)$ (Table 3).

In obese non-diabetic, a positive correlation was found between age and PPBG $(r=0.381, p<0.038)$. BMI was positively correlated with LAR

Table 2: Adipokines and oxidative stress parameters details of the subjects in groups

\begin{tabular}{|c|c|c|c|}
\hline Parameters & Healthy control & Obese non-diabetic & Obese T2DM \\
\hline Leptin (ng/ml) & $6.82 \pm 3.59$ & $25.67 \pm 8.13$ & $26.89 \pm 4.45$ \\
\hline $\mathrm{p}$ & & $<0.001^{*}$ & $<0.001^{*},<0.544^{* *}$ \\
\hline Adiponectin (ng/ml) & $15.53 \pm 3.42$ & $8.93 \pm 1.77$ & $7.56 \pm 1.66$ \\
\hline $\mathrm{p}$ & & $<0.001^{*}$ & $<0.001^{*},<0.079^{* *}$ \\
\hline Leptin/adiponectin ratio & $0.47 \pm 0.27$ & $3.02 \pm 1.38$ & $3.81 \pm 1.38$ \\
\hline $\mathrm{p}$ & & $<0.001^{*}$ & $<0.001^{*},<0.007^{* *}$ \\
\hline AOPP $(\mu \mathrm{mol} / \mathrm{l})$ & $165.35 \pm 41.79$ & $208.84 \pm 42.32$ & $302.67 \pm 57.64$ \\
\hline PC (nmol/mg protein) & $0.72 \pm 0.07$ & $0.98 \pm 0.22$ & $1.46 \pm 0.24$ \\
\hline $\mathrm{p}$ & & $<0.001^{*}$ & $<0.001^{*},<0.001^{* *}$ \\
\hline
\end{tabular}

oxidation of protein products; PC: Protein carbonyl, T2DM: Type 2 diabetic mellitus

Table 3: Spearman's correlation among measured parameters in healthy control

\begin{tabular}{|c|c|c|c|c|c|c|c|c|c|c|c|}
\hline Parameters & Age & BMI & FBG & PPBG & HbA1c & Insulin & HOMAIR & Leptin & Adiponectin & LAR & AOPP \\
\hline \multicolumn{12}{|l|}{ BMI } \\
\hline $\mathrm{r}$ & 0.168 & 1.000 & & & & & & & & & \\
\hline $\mathrm{p}$ & 0.375 & & & & & & & & & & \\
\hline \multicolumn{12}{|l|}{ FBG } \\
\hline$r$ & 0.295 & -0.131 & 1.000 & & & & & & & & \\
\hline $\mathrm{p}$ & 0.113 & 0.491 & & & & & & & & & \\
\hline \multicolumn{12}{|l|}{ PPBG } \\
\hline $\mathrm{p}$ & 0.908 & 0.567 & 0.361 & & & & & & & & \\
\hline \multicolumn{12}{|l|}{$\mathrm{HbA1c}$} \\
\hline $\mathrm{r}$ & 0.230 & -0.237 & 0.099 & 0.240 & 1.000 & & & & & & \\
\hline $\mathrm{p}$ & 0.222 & 0.207 & 0.602 & 0.200 & & & & & & & \\
\hline \multicolumn{12}{|l|}{ Insulin } \\
\hline$r$ & 0.053 & -0.176 & 0.050 & 0.118 & 0.120 & 1.000 & & & & & \\
\hline $\mathrm{p}$ & 0.780 & 0.352 & 0.794 & 0.536 & 0.526 & & & & & & \\
\hline \multicolumn{12}{|l|}{ HOMA-IR } \\
\hline $\mathrm{p}$ & 0.528 & 0.545 & 0.300 & 0.428 & 0.484 & 0.001 & & & & & \\
\hline \multicolumn{12}{|l|}{ Leptin } \\
\hline r & 0.042 & 0.121 & -0.112 & 0.219 & -0.350 & 0.073 & 0.072 & 1.000 & & & \\
\hline $\mathrm{p}$ & 0.826 & 0.523 & 0.555 & 0.244 & 0.058 & 0.700 & 0.707 & & & & \\
\hline \multicolumn{12}{|l|}{ Adiponectin } \\
\hline$r$ & -0.042 & -0.163 & 0.169 & 0.081 & 0.205 & 0.107 & 0.125 & -0.219 & 1.000 & & \\
\hline $\mathrm{p}$ & 0.824 & 0.389 & 0.372 & 0.670 & 0.276 & 0.574 & 0.510 & 0.245 & & & \\
\hline \multicolumn{12}{|l|}{ LAR } \\
\hline $\mathrm{r}$ & 0.070 & 0.228 & -0.130 & 0.186 & $-.383^{*}$ & 0.019 & 0.021 & $0.914^{* *}$ & $-0.547^{* *}$ & 1.000 & \\
\hline $\mathrm{p}$ & 0.712 & 0.226 & 0.492 & 0.325 & 0.037 & 0.921 & 0.914 & 0.001 & 0.002 & & \\
\hline \multicolumn{12}{|l|}{ AOPP } \\
\hline $\mathrm{r}$ & -0.043 & -0.232 & 0.191 & 0.091 & $0.411^{*}$ & 0.180 & 0.204 & -0.069 & 0.265 & -0.189 & 1.000 \\
\hline $\mathrm{p}$ & 0.823 & 0.216 & 0.312 & 0.634 & 0.024 & 0.341 & 0.281 & 0.717 & 0.157 & 0.316 & \\
\hline \multicolumn{12}{|l|}{$\mathrm{PC}$} \\
\hline $\mathrm{r}$ & 0.082 & -0.221 & 0.209 & 0.007 & 0.117 & 0.069 & 0.080 & -0.009 & -0.126 & 0.021 & 0.170 \\
\hline $\mathrm{p}$ & 0.668 & 0.241 & 0.268 & 0.969 & 0.536 & 0.719 & 0.676 & 0.962 & 0.509 & 0.914 & 0.370 \\
\hline
\end{tabular}

*Correlation is significant at the 0.05 level (two-tailed). ${ }^{* *}$ Correlation is significant at the 0.01 level (two-tailed). BMI: Body mass index, FBG: Fasting blood glucose, PPBG: Postprandial blood glucose, HbA1c: Glycated hemoglobin, HOMA-IR: Homeostasis model assessment of insulin resistance, LAR: Leptin/adiponectin ratio, AOPP: Advanced oxidation of protein products, PC: Protein carbonyl 
$(\mathrm{r}=0.375, \mathrm{p}<0.041)$. LAR positively correlated with leptin $(\mathrm{r}=0.909$, $\mathrm{p}<0.001)$ but negatively correlated with adiponectin $(\mathrm{r}=-0.384$, $\mathrm{p}<0.036)$. HOMA-IR positively correlated with FBG $(\mathrm{r}=0.429, \mathrm{p}=0.018)$ and insulin $(\mathrm{r}=0.968, \mathrm{p}=0.001)$. AOPP positive correlation was found among BMI $(\mathrm{r}=0.429, \mathrm{p}<0.018)$ and $\mathrm{PC}(\mathrm{r}=0.702, \mathrm{p}=0.001)$ whereas a negative correlation was found between PC and adiponectin ( $\mathrm{r}=-0.412$, $\mathrm{p}<0.024$ ) (Table 4).

In obese T2DM, FBG positive correlation found among PPBG, HbA1c, insulin, and HOMA-IR $(r=0.502, p<0.005 ; r=0.373, p<0.042 ; r=0.437$, $\mathrm{p}=0.016$; and $\mathrm{r}=0.670, \mathrm{p}=0.001$ ), respectively. HOMA-IR positive correlation found among PPBG, HbA1c, and insulin ( $\mathrm{r}=0.528, \mathrm{p}=0.001$; $\mathrm{r}=0.536, \mathrm{p}=0.002$; and $\mathrm{r}=0.867, \mathrm{p}=0.001$ ), respectively. A negative correlation between leptin and adiponectin $(r=-0.402, p<0.028)$. LAR positively correlated with leptin $(\mathrm{r}=0.731, \mathrm{p}<0.001)$ but negatively correlated with adiponectin $(\mathrm{r}=-0.879, \mathrm{p}<0.001)$. AOPP positively correlated with FBS, PPBG, HOMA-IR, and PC $(\mathrm{r}=0.385, \mathrm{p}<0.036$; $r=0.422, p<0.020 ; r=0.497, p=0.005$; and $r=0.482, p=0.007$ ) whereas PC negatively correlated with adiponectin $(\mathrm{r}=-0.371, \mathrm{p}<0.043)$ (Table 5).

\section{DISCUSSION}

This study was done to examine protein oxidative markers (AOPP, PC) and major metabolic homeostasis markers (adiponectin, leptin, and LAR) in obese subjects with and without T2DM. Obese T2DM and obese non-diabetic had a significantly lower level of serum adiponectin as compared to lean T2DM and healthy control, respectively. Serum adiponectin level was decreased in obese subjects with and without diabetes, a similar trend was also seen in this study [26,27]. Decreased level of adiponectin has been strongly associated with IR and inflammation was seen as the main cause of the link between obesity and T2DM [28]. Adiponectin was negatively correlated with leptin in obese T2DM which is in accordance with previous reports [29]. This was due to increased HOMA-IR and leptin level and decreases adiponectin level which strongly predicts IR [30].

In contrast to adiponectin, leptin levels increase in obesity which is proportional to the degree of adiposity [13,31]. In this study, serum leptin and LAR were higher in both obese T2DM and obese nondiabetic patients with respect to healthy control. From this study, we observed that serum leptin and LAR were higher in obese patients whether obese patients were diabetic or not which is consistent with a previous study $[16,32]$. An earlier report showed that serum leptin was negatively correlated with serum adiponectin; this study also showed similar trends in obese T2DM patients [33]. As reported by Al-Hamodi et al. [13], an inverse association between leptin and adiponectin was observed which highlight the recent findings that indicated that the two adipokines interact with each other in the inflection of T2DM risks. Both leptin and adiponectin play an important role in the maintenance of metabolic homeostasis and fat accumulation in adipose tissue [9]. In central obesity, disturbance of their level leads to increase appetite and reduced energy expenditure results in IR by the accumulation of fat in adipose tissue [1]

In this study, HOMA-IR and LAR both indexes of IR were increased in obese non-diabetic and obese T2DM as compared to healthy control. As suggested by Satoh et al. [34], Kotani et al. [35], and LAR represented as a potential marker of an atherogenic index in obese type 2 diabetic patients. LAR is the most reliable marker of IR than HOMA-IR to leptin or adiponectin alone in obesity with and without type 2 diabetes [30]. It is also reported LAR to be associated with central obesity and metabolic syndrome subjects [35]. In this study, we observed LAR be positively correlated with BMI in obese non-

Table 4: Spearman's correlation among measured parameters in obese nondiabetic

\begin{tabular}{|c|c|c|c|c|c|c|c|c|c|c|c|}
\hline Parameters & Age & BMI & FBG & PPBG & HbA1c & Insulin & HOMA-IR & Leptin & Adiponectin & LAR & AOPP \\
\hline \multicolumn{12}{|l|}{ BMI } \\
\hline $\mathrm{r}$ & -0.204 & 1.000 & & & & & & & & & \\
\hline $\mathrm{p}$ & 0.279 & & & & & & & & & & \\
\hline \multicolumn{12}{|l|}{$\mathrm{FBG}$} \\
\hline$r$ & -0.152 & 0.251 & 1.000 & & & & & & & & \\
\hline $\mathrm{p}$ & 0.422 & 0.180 & & & & & & & & & \\
\hline \multicolumn{12}{|l|}{ PPBG } \\
\hline $\mathrm{p}$ & 0.038 & 0.949 & 0.565 & & & & & & & & \\
\hline \multicolumn{12}{|l|}{ HbA1c } \\
\hline r & -0.187 & 0.217 & 0.149 & 0.307 & 1.000 & & & & & & \\
\hline $\mathrm{p}$ & 0.323 & 0.249 & 0.433 & 0.098 & & & & & & & \\
\hline \multicolumn{12}{|l|}{ Insulin } \\
\hline r & 0.120 & 0.143 & 0.252 & 0.282 & 0.051 & 1.000 & & & & & \\
\hline $\mathrm{p}$ & 0.527 & 0.452 & 0.180 & 0.131 & 0.787 & & & & & & \\
\hline \multicolumn{12}{|l|}{ HOMA-IR } \\
\hline $\mathrm{p}$ & 0.776 & 0.350 & 0.018 & 0.176 & 0.577 & 0.001 & & & & & \\
\hline \multicolumn{12}{|l|}{ LEPTIN } \\
\hline r & 0.095 & 0.287 & 0.185 & -0.173 & 0.109 & 0.187 & 0.183 & 1.000 & & & \\
\hline $\mathrm{p}$ & 0.616 & 0.124 & 0.328 & 0.361 & 0.565 & 0.322 & 0.334 & & & & \\
\hline \multicolumn{12}{|l|}{ Adiponectin } \\
\hline r & -0.127 & 0.018 & -0.049 & -0.287 & -0.319 & -0.078 & -0.106 & -0.116 & 1.000 & & \\
\hline $\mathrm{p}$ & 0.504 & 0.925 & 0.797 & 0.124 & 0.086 & 0.681 & 0.577 & 0.541 & & & \\
\hline \multicolumn{12}{|l|}{ LAR } \\
\hline $\mathrm{r}$ & 0.064 & $0.375^{*}$ & 0.283 & -0.075 & 0.203 & 0.295 & 0.321 & $0.909 * *$ & $-0.384^{*}$ & 1.000 & \\
\hline $\mathrm{p}$ & 0.736 & 0.041 & 0.130 & 0.694 & 0.283 & 0.113 & 0.084 & 0.001 & 0.036 & & \\
\hline \multicolumn{12}{|l|}{ AOPP } \\
\hline $\mathrm{r}$ & -0.006 & $0.429 *$ & -0.059 & 0.151 & 0.165 & -0.151 & -0.105 & 0.025 & -0.312 & 0.196 & 1.000 \\
\hline $\mathrm{p}$ & 0.973 & 0.018 & 0.756 & 0.427 & 0.384 & 0.426 & 0.582 & 0.897 & 0.093 & 0.300 & \\
\hline \multicolumn{12}{|l|}{$\mathrm{PC}$} \\
\hline $\mathrm{r}$ & -0.034 & 0.335 & -0.084 & 0.013 & 0.102 & -0.191 & -0.159 & -0.181 & $-0.412^{*}$ & -0.015 & $0.702^{* *}$ \\
\hline $\mathrm{p}$ & 0.857 & 0.071 & 0.661 & 0.945 & 0.593 & 0.312 & 0.403 & 0.340 & 0.024 & 0.936 & 0.001 \\
\hline
\end{tabular}

${ }^{*}$ Correlation is significant at the 0.05 level (two-tailed), ${ }^{* *}$ Correlation is significant at the 0.01 level (two-tailed). BMI: Body mass index, FBG: Fasting blood glucose, PPBG: Post-prandial blood glucose, HbA1c: Glycated hemoglobin, HOMA-IR: Homeostasis model assessment of insulin resistance, LAR: Leptin/adiponectin ratio, AOPP: Advanced oxidation of protein products, PC: Protein carbonyl 
Table 5: Spearman's correlation among measured parameters in obese type 2 diabetes mellitus

\begin{tabular}{|c|c|c|c|c|c|c|c|c|c|c|c|}
\hline Parameters & Age & BMI & FBG & PPBG & HbA1c & Insulin & HOMA-IR & Leptin & Adiponectin & LAR & AOPP \\
\hline \multicolumn{12}{|l|}{ BMI } \\
\hline $\mathrm{r}$ & -0.075 & 1.000 & & & & & & & & & \\
\hline $\mathrm{p}$ & 0.695 & & & & & & & & & & \\
\hline \multicolumn{12}{|l|}{ FBG } \\
\hline $\mathrm{r}$ & -0.186 & 0.142 & 1.000 & & & & & & & & \\
\hline $\mathrm{p}$ & 0.325 & 0.453 & & & & & & & & & \\
\hline \multicolumn{12}{|l|}{$\begin{array}{l}\mathrm{P} \\
\text { PPBG }\end{array}$} \\
\hline $\mathrm{r}$ & -0.077 & -0.024 & $0.502^{* *}$ & 1.000 & & & & & & & \\
\hline $\mathrm{p}$ & 0.685 & 0.899 & 0.005 & & & & & & & & \\
\hline \multicolumn{12}{|l|}{ HbA1c } \\
\hline $\mathrm{r}$ & -0.204 & 0.114 & $0.373^{*}$ & 0.330 & 1.000 & & & & & & \\
\hline $\mathrm{p}$ & 0.279 & 0.549 & 0.042 & 0.075 & & & & & & & \\
\hline \multicolumn{12}{|l|}{ Insulin } \\
\hline$r$ & -0.242 & 0.071 & $0.437^{*}$ & 0.279 & $0.527^{* *}$ & 1.000 & & & & & \\
\hline $\mathrm{p}$ & 0.197 & 0.711 & 0.016 & 0.136 & 0.003 & & & & & & \\
\hline \multicolumn{12}{|l|}{ HOMA-IR } \\
\hline $\mathrm{r}$ & -0.276 & 0.073 & $0.670^{* *}$ & $0.528^{* *}$ & $0.536^{* *}$ & $0.867^{* *}$ & 1.000 & & & & \\
\hline $\mathrm{p}$ & 0.139 & 0.702 & 0.001 & 0.003 & 0.002 & 0.001 & & & & & \\
\hline \multicolumn{12}{|l|}{ Leptin } \\
\hline $\mathrm{r}$ & 0.191 & 0.062 & -0.070 & -0.138 & -0.032 & -0.209 & -0.216 & 1.000 & & & \\
\hline $\mathrm{p}$ & 0.313 & 0.744 & 0.715 & 0.469 & 0.865 & 0.268 & 0.252 & & & & \\
\hline \multicolumn{12}{|l|}{$\begin{array}{l}\text { p } \\
\text { Adiponectin }\end{array}$} \\
\hline $\mathrm{r}$ & -0.151 & -0.138 & 0.102 & 0.201 & -0.190 & -0.021 & 0.037 & $-0.402 *$ & 1.000 & & \\
\hline $\mathrm{p}$ & 0.427 & 0.467 & 0.593 & 0.287 & 0.315 & 0.914 & 0.848 & 0.028 & & & \\
\hline \multicolumn{12}{|l|}{ LAR } \\
\hline $\mathrm{r}$ & 0.126 & 0.104 & -0.106 & -0.209 & 0.170 & -0.056 & -0.111 & $0.731^{* *}$ & $-0.879 * *$ & 1.000 & \\
\hline $\mathrm{p}$ & 0.509 & 0.586 & 0.577 & 0.268 & 0.369 & 0.768 & 0.559 & 0.001 & 0.001 & & \\
\hline \multicolumn{12}{|l|}{$\mathrm{AOPP}$} \\
\hline$r$ & -0.073 & 0.351 & $0.385^{*}$ & $0.422^{*}$ & 0.317 & 0.321 & $0.497^{* *}$ & -0.201 & -0.045 & -0.029 & 1.000 \\
\hline $\mathrm{p}$ & 0.703 & 0.057 & 0.036 & 0.020 & 0.088 & 0.084 & 0.005 & 0.287 & 0.813 & 0.880 & \\
\hline \multicolumn{12}{|l|}{$\mathrm{PC}$} \\
\hline $\mathrm{r}$ & -0.045 & 0.069 & 0.142 & 0.105 & -0.090 & 0.227 & 0.289 & -0.336 & $-0.371 *$ & 0.084 & $0.482^{* *}$ \\
\hline $\mathrm{p}$ & 0.813 & 0.716 & 0.454 & 0.580 & 0.635 & 0.228 & 0.121 & 0.070 & 0.043 & 0.658 & 0.007 \\
\hline
\end{tabular}

${ }^{*}$ Correlation is significant at the 0.05 level (two-tailed). ${ }^{* *}$ Correlation is significant at the 0.01 level (two-tailed). BMI: Body mass index, FBG: Fasting blood glucose, PPBG: Post-prandial blood glucose, HbA1c: Glycated hemoglobin, HOMA-IR: Homeostasis model assessment of insulin resistance, LAR: Leptin/adiponectin ratio, AOPP: Advanced oxidation of protein products, PC: Protein carbonyl

diabetic such relation was also seen in postmenopausal women with metabolic syndrome [36]. In previous studies, it was also found that HOMA-IR and LAR have a significant positive correlation [36]. However, this study does not find such relation this might be due to a small sample population studied. IR state is linked to chronic lowgrade inflammation in hypertrophied adipose tissue that promotes the initiation and progression of metabolic disorders and T2DM.

It is well studied that obesity induces OS that plays an important role in the pathogenesis of diabetes. AOPP had a significantly higher level in obese T2DM and obese non-diabetic in contrast to lean healthy control. AOPPs increased level is reported to be associated with obesity and diabetes [20,37]. Increased AOPPs may be an independent risk factor for coronary artery disease [19] and early marker of endothelial dysfunction in T2DM patients [38]. In this study, AOPP positively correlated with BMI, PC in obese non-diabetic subjects and with FBG, PPBG, HOMA-IR, and PC in obese T2DM which was in accordance with previous studies [39]. Li et al. [40] also reported that at 32-36 weeks gestational diabetes mellitus showed a significant relationship between AOPP and FBG level compared to normal pregnancies. Increase in levels of AOPP and PC may lead to development in the growth of diabetic complications and cardiovascular diseases.

In this study, we found a significant increase in PC level in obese T2DM patients as compared to obese non-diabetic and healthy control which was in accordance with previous reports [41,42]. According to Frohnert et al. [43], human obesity OS is linked to protein carbonylation which is directly correlated to adiposity and serum free fatty acids that may eventually lead to the development of IR. Studies have shown that in aging proteome accumulated OS-related post-translational modification including glycation, lipid peroxidation, and carbonylation observed in chronic inflammation, metabolic syndrome, and diabetes [44]. This study found an inverse correlation with increased PC level and decreased adiponectin in obese non-diabetic and obese T2DM which is in accordance with previous studies [45]. Therefore, this study supports the relation of obesity between protein OS markers and circulating adipokines.

\section{CONCLUSION}

Present the study suggests that an increase in levels of leptin, insulin, AOPP, and PC in obese non-diabetic subjects and obese T2DM patients play an important role in reducing the level of adiponectin. A significant increase in the level of HOMA-IR and LAR in obese suggests in the development of T2DM and IR. Moreover, obese subjects have increased OS that may contribute to the dysregulation of adipokines and altered glucose metabolism.

\section{ACKNOWLEDGMENT}

Authors are grateful to UIET (Branch Biotechnology), Panjab University, Chandigarh, India, and I. K. Gujral Punjab Technical University, Jalandhar, Punjab, India, for providing infrastructure and facilities to carry out this research work.

\section{DISCLOSURE STATEMENT}

The authors declare that there are no conflicts of interest.

\section{REFERENCES}

1. Matsuda M, Shimomura I. Increased oxidative stress in obesity: Implications for metabolic syndrome, diabetes, hypertension, dyslipidemia, atherosclerosis, and cancer. Obes Res Clin Pract 2013;7:e330-41. 
2. Greenberg AS, Obin MS. Obesity and the role of adipose tissue in inflammation and metabolism. Am J Clin Nutr 2006;83:461S-465S

3. Nakanishi S, Yamane K, Kamei N, Nojima H, Okubo M, Kohno N, et al. A protective effect of adiponectin against oxidative stress in Japanese Americans: The association between adiponectin or leptin and urinary isoprostane. Metabolism 2005;54:194-9.

4. Blüher M, Mantzoros CS. From leptin to other adipokines in health and disease: Facts and expectations at the beginning of the $21^{\text {st }}$ century. Metabolism 2015;64:131-45.

5. Yadav A, Kataria MA, Saini V, Yadav A. Role of leptin and adiponectin in insulin resistance. Clin Chim Acta 2013;417:80-4.

6. Yamauchi T, Kadowaki T. Adiponectin receptor as a key player in healthy longevity and obesity-related diseases. Cell Metab 2013;17:185-96.

7. Aleidi S, Issa A, Bustanji H, Khalil M, Bustanji Y. Adiponectin serum levels correlate with insulin resistance in Type 2 diabetic patients. Saudi Pharm J 2015;23:250-6.

8. Shibata R, Murohara T, Ouchi N. Protective role of adiponectin in cardiovascular disease. Curr Med Chem 2012;19:5459-66.

9. Stern JH, Rutkowski JM, Scherer PE. Adiponectin, leptin, and fatty acids in the maintenance of metabolic homeostasis through adipose tissue crosstalk. Cell Metab 2016;23:770-84.

10. Saad EA, Habib SA, Refai WA, Elfayoumy AA. Malondialdehyde, adiponectin, nitric oxide, $C$ reactive protein, tumor necrosis factoralpha and insulin resistance relationships and inter-relationships in Type 2 diabetes early stage is metformin alone adequate in this stage. Int J Pharm Pharm Sci 2017;9:176-81.

11. Yamauchi T, Kamon J, Waki H, Terauchi Y, Kubota N, Hara K, et al. The fat-derived hormone adiponectin reverses insulin resistance associated with both lipoatrophy and obesity. Nat Med 2001;7:941-6.

12. Iikuni N, Lam QL, Lu L, Matarese G, La Cava A. Leptin and inflammation. Curr Immunol Rev 2008:4:70-9.

13. Al-Hamodi Z, Al-Habori M, Al-Meeri A, Saif-Ali R. Association of adipokines, leptin/adiponectin ratio and C-reactive protein with obesity and Type 2 diabetes mellitus. Diabetol Metab Syndr 2014;6:99.

14. Farooqi IS, O'Rahilly S. Leptin: A pivotal regulator of human energy homeostasis. Am J Clin Nutr 2009;89:980S-984S.

15. Chen VC, Chen CH, Chiu YH, Lin TY, Li FC, Lu ML, et al. Leptin/ adiponectin ratio as a potential biomarker for metabolic syndrome in patients with schizophrenia. Psychoneuroendocrinology 2018:92:34-40.

16. Hamed EA, Zakary MM, Ahmed NS, Gamal RM. Circulating leptin and insulin in obese patients with and without Type 2 diabetes mellitus: Relation to ghrelin and oxidative stress. Diabetes Res Clin Pract 2011; 94:434-41

17. Fernández-Sánchez A, Madrigal-Santillán E, Bautista M, EsquivelSoto J, Morales-González A, Esquivel-Chirino C, et al. Inflammation, oxidative stress, and obesity. Int J Mol Sci 2011;12:3117-32

18. Witko-Sarsat V, Friedlander M, Nguyen Khoa T, Capeillère-Blandin C, Nguyen AT, Canteloup S, et al. Advanced oxidation protein products as novel mediators of inflammation and monocyte activation in chronic renal failure. J Immunol 1998;161:2524-32.

19. Kaneda H, Taguchi J, Ogasawara K, Aizawa T, Ohno M. Increased level of advanced oxidation protein products in patients with coronary artery disease. Atherosclerosis 2002;162:221-5.

20. Piwowar A, Knapik-Kordecka M, Warwas M. AOPP and its relations with selected markers of oxidative/antioxidative system in Type 2 diabetes mellitus. Diabetes Res Clin Pract 2007;77:188-92.

21. Reznick AZ, Packer L. Oxygen radicals in biological systems Part C. Methods Enzymol 1994;233:357-63.

22. Gelisgen R, Genc H, Kayali R, Oncul M, Benian A, Guralp O, et al. Protein oxidation markers in women with and without gestational diabetes mellitus: A possible relation with paraoxonase activity. Diabetes Res Clin Pract 2011;94:404-9.

23. Atabek ME, Keskin M, Yazici C, Kendirci M, Hatipoglu N, Koklu E, et al. Protein oxidation in obesity and insulin resistance. Eur J Pediatr 2006; $165: 753-6$

24. Friedewald WT, Levy RI, Fredrickson DS. Estimation of the concentration of low-density lipoprotein cholesterol in plasma, without use of the preparative ultracentrifuge. Clin Chem 1972;18:499-502.

25. Matthews DR, Hosker JP, Rudenski AS, Naylor BA, Treacher DF, Turner RC, et al. Homeostasis model assessment: Insulin resistance and beta-cell function from fasting plasma glucose and insulin concentrations in man. Diabetologia 1985;28:412-9.

26. Salah A, Ragab M, Mansour W, Taher M. Leptin and adiponectin are valuable serum markers explaining obesity bronchial asthma interrelationship. Egypt J Chest Dis Tuberc 2015;64:529-33.

27. Hong SB, Lee JJ, Kim SH, Suh YJ, Han JY, Kim YS, et al. The effects of adiponectin and inflammatory cytokines on diabetic vascular complications in obese and non-obese patients with Type 2 diabetes mellitus. Diabetes Res Clin Pract 2016;111:58-65.

28. Esser N, Legrand-Poels S, Piette J, Scheen AJ, Paquot N. Inflammation as a link between obesity, metabolic syndrome and Type 2 diabetes. Diabetes Res Clin Pract 2014:105:141-50.

29. Fernández-Real JM, Botas-Cervero P,López-BermanoA, Casamitjana R, Funahashi T, Delgado E, et al. Adiponectin is independently associated with glycosylated haemoglobin. Eur J Endocrinol 2004;150:201-5.

30. Inoue M, Yano M, Yamakado M, Maehata E, Suzuki S. Relationship between the adiponectin-leptin ratio and parameters of insulin resistance in subjects without hyperglycemia. Metabolism 2006;55:1248-54.

31. Ali SH, Al-nuaimi AM, Al-musawi BJ. Serum irisin and leptin levels in obese and non-obese women with polycystic ovary syndrome with reference to glucose homeostasis. Int J Pharm Pharm Sci 2016;8:276-83.

32. Stefanović A, Kotur-Stevuljević J, Spasić S, Bogavac-Stanojević N, Bujisić N. The influence of obesity on the oxidative stress status and the concentration of leptin in Type 2 diabetes mellitus patients. Diabetes Res Clin Pract 2008:79:156-63.

33. Matsubara M, Maruoka S, Katayose S. Inverse relationship between plasma adiponectin and leptin concentrations in normal-weight and obese women. Eur J Endocrinol 2002;147:173-80

34. Satoh N, Naruse M, Usui T, Tagami T, Suganami T, Yamada K, et al. Leptin-to-adiponectin ratio as a potential atherogenic index in obese Type 2 diabetic patients. Diabetes Care 2004;27:2488-90.

35. Kotani K, Sakane N, Saiga K, Kurozawa Y. Leptin: Adiponectin ratio as an atherosclerotic index in patients with Type 2 diabetes: Relationship of the index to carotid intima-media thickness. Diabetologia 2005; 48:2684-6

36. Gupta V, Mishra S, Mishra S, Kumar S, Gupta V. Association of leptin: Adiponectin ratio and metabolic risk markers in postmenopausal women. Immunol Lett 2018;196:63-7.

37. Krzystek-Korpacka M, Patryn E, Boehm D, Berdowska I, Zielinski B, Noczynska A, et al. Advanced oxidation protein products (AOPPs) in juvenile overweight and obesity prior to and following weight reduction. Clin Biochem 2008;41:943-9.

38. Liang M, Wang J, Xie C, Yang Y, Tian JW, Xue YM, et al. Increased plasma advanced oxidation protein products is an early marker of endothelial dysfunction in Type 2 diabetes patients without albuminuria 2. J Diabetes 2014;6:417-26

39. Koçak H, Oner-Iyidoğan Y, Gürdöl F, Oner P, Süzme R, Esin D, et al. Advanced oxidation protein products in obese women: Its relation to insulin resistance and resistin. Clin Exp Med 2007;7:173-8

40. Li H, Yin Q, Li N, Ouyang Z, Zhong M. Plasma markers of oxidative stress in patients with gestational diabetes mellitus in the second and third trimester. Obstet Gynecol Int 2016;2016:3865454.

41. Pandey KB, Mishra N, Rizvi SI. Protein oxidation biomarkers in plasma of Type 2 diabetic patients. Clin Biochem 2010;43:508-11

42. Bollineni RC, Fedorova M, Blüher M, Hoffmann R. Carbonylated plasma proteins as potential biomarkers of obesity induced Type 2 diabetes mellitus. J Proteome Res 2014;13:5081-93.

43. Frohnert BI, Sinaiko AR, Serrot FJ, Foncea RE, Moran A, Ikramuddin S, et al. Increased adipose protein carbonylation in human obesity. Obesity (Silver Spring) 2011;19:1735-41

44. Perkins BA, Rabbani N, Weston A, Ficociello LH, Adaikalakoteswari A, Niewczas M, et al. Serum levels of advanced glycation endproducts and other markers of protein damage in early diabetic nephropathy in Type 1 diabetes. PLoS One 2012;7:e35655

45. Indulekha K, Surendar J, Anjana RM, Gokulakrishnan K, Balasubramanyam M, Aravindhan V, et al. Circulating levels of high molecular weight (HMW) adiponectin and total adiponectin in relation to fat distribution, oxidative stress and inflammation in asian Indians. Dis Markers 2012;33:185-92 